\title{
La Lingua Albanese della Pubblicità
}

\section{Doi:10.5901/jesr.2014.v4n4p241}

\author{
Svjetllana Titini
}

\begin{abstract}
The advertising is an omnipresent and omnipotent reality of everyday life. This article will point out the characteristics of the Albanian language of advertising. First, it will be highlighted that use of the language of advertising is taking advantage of the common language and emphasizing the expressive possibilities of the last one.Later, it will be shown the creation of innovations from the use of the technique of advertising language both at the level of microstructures and macrostructures that give rise to a multiple relationship with the natural language and to the common enrichment.
\end{abstract}

Keywords: lingua albanese della pubblicità, messaggio pubblicitario, lingua artificiale, linguaggio della pubblicità.

\section{Introduzione}

La pubblicità è ormai parte della vita dell' uomo nella società moderna. Essa rappresenta un importante forma di comunicazione tra chi promuove idee, beni o servizi e il pubblico a cui si rivolge il messagio attraverso sempre più strumenti pubblicitari classici e inovativi.

La grande crescità della pubblicità nel corso degli anni e il suo essere un fenomeno complesso ha reso la pubblicità, nei suoi vari aspetti, oggetto di studio nell' interesse di una pluralità di discipline.

Sono numerosi gli studi che hanno indagato anche sull'aspetto linguistico della pubblicità. L'analisi del rapporto tra lingua e pubblicità si basa su una triplice prospettiva, ciascuna mirante a gettar luce su una specifica dimensione: la pubblicità come lingua creata artificialmente per uno scopo, la pubblicità come strumento che usa il codice della lingua e la pubblicità come arte di trasformazione della lingua naturale.

Qundi abbiamo portato brevemente alcuni cenni introduttivi sull'argomento prima di affrontare concretamente l'analisi della lingua albanese della pubblicità.

II linguaggio pubblicitario è una sintesi di diversi codici - visivo, verbale, oggettuale, sonoro e gestuale - tra cui emerge il ruolo essenziale della componente linguistica di unificare tutti gli elementi della comunicazione pubblicitaria e di dare luogo alla comprensibilità del messaggio pubblicitario specificando il significato dell'immagine.

La pubblicità parla la stessa lingua del paese in cui essa viene diffusa ${ }^{1}$. Mentre utilizza la lingua comune - che al suo interno comprende diversi sottocodici impiegati in funzione del tipo di prodotto da promuovere - il linguaggio della pubblicità, con le sue proposte, cerca di sedurre e di convincere il pubblico attraverso l'uso di vocaboli nuovi e inediti o la ricontestualizzazione di forme linguistiche usuali.

In questo contesto tra la lingua della pubblicità e la lingua comune si instaura un rapporto di continuo scambio sul quale gli studiosi hanno adottato due atteggiamenti opposti: da un lato c'è chi esalta il dinamismo, la creatività e l'immediatezza, caratteristiche tipiche, del linguaggio della pubblicità che sfrutta ed accentua le potenzialità espressive della lingua comune e lo rende più agile, funzionale e moderna; dall' altro lato c'è chi avverte nella produzione ininterrota di parole-merci e l'uso di sintassi parattatica nel linguaggio pubblicitario la causa dell'impoverimento e dell' uniformità della lingua comune.

Detto ciò, la lingua è lo strumento principale della pubblicità che viene utilizzata, modellata e manipolata- a livello delle microstrutture e delle macrostrutture espressive - con sottile abilità di trasmettere un "messaggio" semantico attraverso una innovazione linguistica condivisibile potenzialmente, da tutti i target di pubblico. Dunque la lingua è la chiave di lettura dei segnali inviati e la sua creatività è il punto di forza per provocare la convinzione o la reazione attiva nel ricevente.

\section{Lingua della Pubblicità e Lingua Comune: I Rapporti Nella Pubblicità Albanese}

La pubblicità albanese compare relativamente tardi ed ha riconosciuto una evoluzione frammentaria a causa degli eventi

\footnotetext{
1 In pratica è una tendenza ben nota la codifcazione della comunicazione pubblicitaria in una lingua diversa da quella che parlano $i$ destinatari del mesaggio pubblicitario. L'impiego della lingua straniera è una scelta per motivi diversi che può investire solo lo slogan o tutto il messagio pubblicitario. In quest' ultimo caso non sempre la pubblicità viene susseguita da sottotitoli nella lingua nazionale.
} 
storici del passato. La storia della pubblicita albanese, nel periodo dopo gli anni ' 90 , rispecchia il progressivo sviluppo e l'affermazione del linguaggio della pubblicità.

II presente contributo prende inizio da un duplice ordine di considerazioni: la prima che ha dato spunto alla nostra ricerca è stata l'idea di analizzare se la lingua albanese della pubblicità rispecchia delle trasformazioni originali, inedite della lingua come è stato dimostrato dagli studi su altre lingue e quali sono le sue caratteristiche; la seconda è stato il voler evidenziare il panorama delle lingue adoperate nel testo pubblicitario e i valori di riferimento della pubblicità albanese.

II corpus dei dati sul quale si è svolta la nostra analisi raccoglie spot pubblicitari trasmessi nei due canali della rete pubblica TVSH e nei vari canali delle emittenti private. Inoltre a questi si aggiungono anche dati di pubblicità raccolti dalla stampa e dalla pubblicità outdoor ${ }^{2}$. I dati al interno del corpus interessano un ampia varietà di categorie merceologiche $\mathrm{e}$ di prodotti.

Come si è accennato la tecnica del linguaggio pubblicitario tenta attraverso le insistenti invenzioni linguistiche di produrre stupore e straniamento nel ricevente con l'obiettivo finale di indurrlo a comprare.

Nella lingua naturale l'uso degli affissi serve non solo al continuo arrichimento del lessico ma accentua una delle tante possibilità espressive della lingua. Nella lingua della pubblicità albanese si nota l'uso incrementato di prefissi di origine greca e latina super-, mini-, maxi-, ultra-, mega-, mikro-. Essi svolgono la funzione di intensificatori che contribuiscono ad amplificare o graduare il significato della parola: superoferta, superkrokante, superpërthithëse, supersexy, superkurseni, minikruasan, minikotoleta, ministrudel, ultrathithëse, megafestë, mikrokapsulë, mikrokristale. Nella lingua della pubblicità i prefissi maxi, mini, extra, mega vengono utilizzati non solo in aggiunta ad una parola preesistente ma anche autonomamente in funzione nominale ed aggettivale. (E disponueshme në madhësitë Normal, Super, dhe Maxi; vjen në format Max; një karakteristikë ekstra; mbështetje extra; garanci ekstra; Madhësi Mini; Oferta Mega, është super ). Le suddette parole nelle combinazioni sintagmatiche sono collocate dietro la parola con cui si trovano in rapporto sintattico super invece può instaurare un rapporto sintagmatico con una parola che lo precede o lo sussegue: super e rehatshme, super e shijshme, super e shpejtë, super e përshtatshme, super e lehtë, Të gjithë ne besojmë në KMY super; E disponueshme në madhësitë Normal dhe Super; Paketa mujore Vodafone club absolutisht super; Telefona smart, çmime super.

Negli ultimi anni si nota il trasferimento dell'utilizzo autonomo dei prefissi dal linguaggio pubblicitario alla lingua comune trovando largo spazio specialmente nel linguaggio giovanile.

La lingua della pubblicità impiega ampiamente gli aggettivi quale categoria di parole che nella lingua albanese sono dotate di valore espressivo o lo possono acquisire facilmente durante il loro uso.(Thomai 2010) Due sono i gruppi di aggettivi il cui uso si prospetta di essere privilegiato nella pubblicità albanese per stordire il destinatario ampliando la risonanza emotiva e contenutistica del testo: il primo gruppo comprende degli aggettivi strutturalmente diversi che non hanno variazione di grado o che hanno un intenso valore espressivo: absolut, ideal, unik, special, perfekt, superior, maksimal, ekstrem, i jashtëzakonshëm, magjik, i përsosur, i shkëlqyer, i mrekullueshëm, i përkryer, magjepsës, tundues, joshës ecc; il secondo gruppo comprende degli aggetivi derivati, formati con l'affisso pa-: i pakrahasueshëm, i papërsëritshëm, të parezistueshme, të pabesueshme, i pandalshëm, i paharrueshëm, i pazëvendësueshëm, e pazhurmshme ecc. Semanticamente questo gruppo di aggettivi esprime una qualità posseduta dal prodotto nel massimo grado rendendo superfluo il confronto con qualsiasi altro prodotto simile. Un sottogruppo degli aggettivi formati con il prefisso -pa identificano caratteristiche negative che vengono a meno grazie al prodotto reclamizzato: e pamundur, të parehatshëm, të padëshirueshme.

L'intensità espressiva delle parole viene rinforzata anche attraverso l'utilizzo delle particelle e degli avverbi che le accompagnano. Questa naturale tendenza della lingua albanese viene moltiplicata nella lingua della pubblicità: më energjike, sa më të favorshme, vaktet më të ushqyeshme, shijet më të preferuara të kohës, brandi më rinor dhe më i modës, kaq i vogël, kaq i madh, aq e vlefshme, shumë të holla, shumë i mirë, shumë cilësor.

La produttività del suffisso -(i)sht nella formazione degli avverbi di maniera nella lingua albanese aumenta nella lingua della pubblicità che fa largo uso di questo gruppo di avverbi: mbështet butësisht, provuar shkencërisht, kërcen mbrapsht, i pranuar ekologjikisht, testuar dermatologjikisht, i testuar klinikisht, lehtësisht të përdorshëm, ndikon negativisht, i akredituar institucionalisht, eksluzivisht për klientët, e fik automatikisht, të programuara gjenetikisht, do të përdorni muskujt tuaj më intensivisht, janë të izoluara elektrikisht, mesatarisht të fortë, thekson ekstremisht qerpikët, lehtësisht të zëvendësueshëm, të ndryshon rrënjësisht jetën. Inoltre nella lingua della pubblicità si osserva una

\footnotetext{
2 Sono raccolti spots pubblicitari trasmessi nei principali canali delle emittenti private: Top Channel, TvKlan, Vizion plus, Albanian Screen, News 24, Oranews, A1Report, abcnews. I dati relativi alla pubblicità scritta sono raccolti da due fonti, giornali e riviste: Gazeta shqiptare, Shqiptarja.com, Panorama, Shekulli, Dita, MAPO, Klan, JAVA e pubblicità outdoor nella città di Tirana.
} 
propensione ad usare gli avverbi formati col suffisso -(i)sht nel costruire il superlativo assoluto degli aggettivi per esprimere l'intensità massima della qualità: përsosmërisht të pastër, absolutisht super, absolutisht fantastike, tmerrësisht efektive, krejtesisht natyral, jashtëzakonisht të dobishme ecc.

L'uso dei fraseologismi, per la loro natura particolarmente espressiva, conferisce al linguaggio pubblicitario una forte carica emozionale ed espressiva favorendo il raggiungimento del suo scopo che è proprio quello di stupire e di attrarre il destinatario suscitando in esso una serie di reazioni: stordimento, divertimento, partecipazione 0 approvazione: shefi nuk më mbyt më në punë -Kit Kat; darka juaj do të mbahet mend; ju mund të rrini duarkryq ecc.

II rapporto scambievole tra la lingua della pubblicità e la lingua comune si riflette chiaramente a livello lessicale nell'ambito di neoformazioni e di divulgazione. La lingua della pubblicità contribuisce a creare nuove parole sul modello della lingua comune (che a volte hanno vita breve e a volte entrano a far parte a tutti gli effetti nel lessico comune) ma il suo contributo si estende nell' ampliare il lessico dei parlanti attraverso la diffusione dei neologismi e del flusso lessicale proveniente da diversi sottocodici (i linguaggi settoriali o puo essere considerato un sottocodice l'uso dei dialetti) che impiega nella comunicazione pubblicitaria.

La lingua della pubblicità propone e fa uso di neologismi coniati: a) per derivazione sulla base di morfemi lessicali gia esistenti nella lingua albanese e altri che sono entrati nel corpo lessicale dai '90 in poi: kundër-trysni, vetajrosës, të çokollatuar, shijues, gjysëmfurçë, të çertifikuar, të patentuar, stabilizues, të personalizuar, ondeluar, tonifikim, detoksifikim, stiloj, filetim, miksoj, arredim, i dizenjuar, e disponueshme, relaksoj, relaksues, dermatologjikisht, i riciklueshëm, miksim, i afetuar, i konfeksionuar, i sofistikuar ecc; b) con l'aggiunta di un significato nuovo a parole esistenti per influsso di una lingua straniera. L'aggetivo anatomik ha il significato: 1.Relativo all'anatomia; il suo nuovo significato è: modellato secondo la forma del corpo umano o di una sua parte, me dizenjo anatomike; II sostantivo ngjitëse ha due significati: 1.Attrezzatura per saldare due parti metalliche, 2 . Sostanza che attacca due superfici poste a contatto; il nuovo significato è: oggetto fornito di speciali sostanze che consentono un'incollatura per contatto, ngjitëse anësore elastike. Altre volte il significato nuovo di una parola esistente è risultato di un processo di trasvalorizzazione semantica che sulla base di associazioni di significato rende possibile il loro uso per denominare nuovi prodotti: kuqalashe è un genere di birra, plus è un operatore celulare, tropikal è un genere di bevanda, popullor è un tipo di salsiccia.

Nel lessico della pubblicità non mancano le parole composte che possono essere denominazioni di prodotti 0 servizi, possono indicare delle qualità 0 di rado designare un processo: kartëpecetë, një pelenë mbathje moderne, Shërbimi Duakredi, AMCkarta është zgjidhja për ju ..., shkumëformuese, mbresëlënëse, jetëndryshuese, gjidhënie.

Nella lingua della pubblicità accanto all' unione delle parole diverse in un unica parola si nota altresì il fenomeno contrario della scomposizione delle parole proprio per impressionare il destinatario. (Kap mot ivi $\mathrm{n}$, shijo ivi; Every day Cotton-Fresh, edhe Cotton, edhe Fresh).

L'abbreviazione delle parole e gli acronimi affollano la lingua della pubblicità per effetto del tempo e dello spazio a disposizione sempre più ridotto nel mercato della pubblicità. Nella maggior parte si tratta di denominazioni di vari brand: info (informazione), tel (telefono), net (internet), çoko (çokollatë); DIGITALB, DIGIGOLD, ALBTV, ABISSNET, ABCOM, EUROSIG. SICRED, ALBtelecom ecc.

Si puo menzionare anche qualche nuova formazione particolare che unisce nella sua struttura numeri e lettere: shërbimi 3N1 (3 në 1)

Negli ultimi anni hanno visto la luce anche molti sintagmi lessicali che sono denominazioni di prodotti vari: frankfurter viçi, koh viçi, letër kuzhine, letër higjenike, makarona orizi, peceta ditore, peceta mujore, makineri përzjerëse, njësi manikyriste, tharëse flokësh, mashë per flokët, tigan elektrik, mixer dore ecc. Un particolare gruppo costituiscono i sintagmi costruiti dalle diverse combinazione di acronimi, parole in lingua albanese e prestiti: AMC Relaks, AMC Spring, AMC Super Universal Mujore, Vodafone Club, Vodafone Card ecc.

II ricorso nei testi pubblicitari all'uso dei prestiti e tecnicismi costituisce una strategia persuasoria meno appariscente ma efficacce che esalta il prodotto senza correre il rischio di suscitare perplessità nel destinatario. I tecnicismi rafforzano nel destinatario del messaggio pubblicitario l'affidabilità nella qualità del prodotto ma contemporaneamente il destinatario si sente stimato nella comunicazione che si instaura con chi propone il messaggio perchè viene riconosciuta la sua capacità di decriptare questo codice (Sergio, 2007). Nella lingua della pubblicità i tecnicismi che derivano da vari settori specialistici dalla tecnologia alla medicina, dalla moda alla gastronomia, dalla politica allo sport vengono adoperati non solo per l' autenticazione scentifica del prodotto in relazione all' appartenenza ad un determinato settore merceologico ma spesso perchè conferiscono al linguaggio pubblicitario una risorsa per differenziare il prodotto e annunciare la sua superiore qualità agli occhi del consumatore.

Una parte considerevole dei tecnicismi usati sono dei prestiti: dizajn, dizajner, interaktivitet, pocorn, timeshift, wifi, aplikacion, check- up, turbo, antiseptik, antimikrobial, analgjezik, make-up, termosensor, detoks, stres, detoksifikim ecc. 
I prestiti non costituiscono l'unica presenza delle lingue straniere nel linguaggio della pubblicità. Spesso la pubblicità communica col pubblico in lingua straniera. Questo tipo di pubblicità si differenzia per la scelta della lingua con cui si rivolge al pubblico, per i componenti della pubblicità che comprende questa scelta comunicativa e infine per le tipologie di prodotti reclamizzati.

Nella pubblicità albanese l'uso della lingua straniera prevale negli slogan pubblicitari invece si rivela limitata il suo impiego per l'intero comunicato commerciale negli spot pubblicitari e nella pubblicità scritta ${ }^{3}$.

La lingua della pubblicità avverte sempre di più la tendenza di avvalersi delle figure retoriche per accentuare la connotazione e l'espressività del messaggio. Nel realizzare la funzione conativa e quella retorico -suasiva i testi pubblicitari in lingua albanese hanno privilegiato l'uso di enunciati ellitichi che non sono semplici frasi affermative ma implicitamente esse proclamano la supremazia dei prodotti sul mercato: Vodafone Komuniteti nr. 1 në Shqipëri; Gjithmonë me ju Deka; Stela Birra ime; Oranews Të parët për lajmin e fundit; Besimi juaj, detyrimi jonë Vienna Insurance Group; Credins on line banka në dorën tuaj; EM Zgjedhja ime e parë; Global Kem Rrezatojmë ambjentin ecc. Nella lingua della pubblicità vengono largamente usati anche gli enunciati enumerativi per il valore descrittivo ed argomentativo che assume l'elenco delle caratteristiche nel creare il buon immagine del prodotto e nel renderlo apprezzabile: Pastërti e përkryer, bardhësi absolute, mbrojtje e ngjyrave - I shkëlqyer Deus; Kripë deti e imët, e jodizuar, e bardhë - Marina; Ha pi, përtyp orbit; Nescafe classic 3 në 1- Kafe, qumësht, sheqer në një. Ne nuk jemi as e para, as më e madhja, as më e vjetra, por jemi më e sigurta- Albsig; Filozofia jone bazohet te shprehja:Sherbim, angazhim, garanci, art - Shaga shpk ecc. A volte l'elenco delle parole si colloca in un crescendo di intensità espressiva che da luogo alla figura retorica del climax: Ibriku pasurues i Ujit, Pure Aqua nga Delimano - I mirë për shëndetin tuaj, portofolin tuaj dhe për gjithë planetin. Un altro procedimento linguistico a cui ricorre constantemente il discorso pubblicitario è la ripetizione alla quale si riferiscono altre forme retoriche distinte tra di loro ma che tutte gli accomuna la funzione di insistere sul concetto. In questo modo attraverso l'insistente ripetizione di parole o espressioni simili il testo pubblicitario intende richiamare alla memoria il prodotto e sollecitare nel consumatore il suo gradimento. La pubblicità risulta carica di esempi di anafora e epifora: Më shumë fanta, më shumë argëtim; Megatek Bëje mirë. Bëje vetë; 125 vite coca cola, 125 vite dhurojmë lumturi; mirë për ju mirë për Shqipërinë BKT; që objekti i duhur t'i ofrohet blerësit të duhur në kohën e duhur Real estate Immobiliari; Ju garantojmë pjesën e duhur në çmimin e duhur Japan Auto Goni; anadiplosi: kur cilësia më e mirë përqafon shijen unike lind një eksperiencë e re, eksperiencë që do të doja ta përjetoj çdo ditë EHW; parallelismo: Artistë në cilësi, mjeshtër në leverdi Conad.

Particolari coloriture espressive conferiscono al linguaggio pubblicitario le espressioni figurate sulla base del trasferimento del significato. Esempi di uso cospicuo delle figure del pensiero nel linguaggio pubblicitario sono: le metafore e sinestesie: jo më flokë të thyer (Shauna Shampo); zbardhëm ëndrrat, ngjyrat e kujtimeve (Deka); kredi e gjelbër (BKT); banka në dorën tuaj, merre bankën me vete (Credins); çmenduni pas blerjeve ..(QTU); Shënoni me interesat më të ulëta në treg (Raiffeisen Bank); Vallëzo me kohën tënde( Eagle Mobile); aromë e ëmbël, që zgjat (Baby Care); le metonìmie: Ku është AIA ka gëzim; 7DAYS MAX ruan freskinë dhe shijen duke lejuar që ju ta shijoni atë edhe kur jeni duke ecur, kurdo kudo; Gjej gol në tapën e kuqalashes; Fëmijet e lumtur përdorin Molfix; Tani që zbulova Lordes mund të dhuroj lule sa herë të dual; gli antitesi: shpenzo pak komuniko shumë Plus; Kaloni të dielat tuaja me një shqetësim më pak dhe një kursim më shumë Taçi Oil; Shumë kërkesa, por vetëm një mënyrë për t'i plotësuar ato menjëherë Societe Generale Albania, Madhësi Mini për rehati Maksimale Everyday; Shumë të shijshme dhe me pak kalori! Aktive brenda, duket nga jashtë- Activia; Ne krijojmë standardet të tjerët na ndjekin Edilplus ecc.; le antonomasie: Të shijojmë së bashku 64 sfida të nxehta nga atdheu i sambas; el derbi Real Madrid -Atletico Madrid: Kush do të jetë "Zorro" i Europës ecc.

Per raggiungere il suo obiettivo di attrarre il consumatore la pubblicità albanese ricorre ampiamente non soltanto alle parole nuove 0 all' uso figurato delle parole ma anche al non uso delle parole. II tacere volontario di ciò che si potrebbe 0 dovrebbe dire nel testo pubblicitario (reticenza) scaturisce nel pubblico grande curiosità e interesse a immaginare il seguito coinvolgendolo attivamente. AMC ...bota jonë ti; shqiptarja.com ...origjina e lajmit; Kurseni që tani për r pushimet verore... ABCOM; Perfekte si ti...Albtelecom; Në shtë pinë tonë kë të vit do të jemi një më shumë ... Spitali

\footnotetext{
${ }^{3}$ Dai dati analizatti sono soltanto alcuni gli spot pubblicitari trasmessi in televisione in lingua straniera accompagati sempre da sottotitoli nella lingua albanese. Questi sono degli spot del settore di telefonia (gli spot di James Belushi per Eagle Mobile), del settore alimentare( lo spot di Antonio Banderas per Orbit) ed uno spot sulla sensibilizzazione sociale( A1 report). Mentre risultano più frequenti $i$ casi di ricorso all'uso della lingua straniera nella pubblicità scritta presente in riviste, giornali e outdoor. Nella pubblicità televisiva si avverte in un caso anche il fenomeno contrario cioè l'accompagnare il testo nella lingua albanese con sottotitoli in lingua straniera (Eagle Mobile). Lo stesso fenomeno si incontra anche nella pubblicità scritta. (Produkte çeliku-steel products/llamarina dhe lama-steel plates \& flats/ trarw dhe profile-steel beams \& SHS..........)
} 
Amerikan; Tekonologjitë e reja nuk janë thjesht produkte të inovacionit... DIGITALB ecc.

Nell'enfatizzare il suo linguaggio spesso la pubblicità si avvale delle figure del suono che donano ai slogan la musicalità e gli rendono piacevoli per le orecchie ed efficaci a coinvolgere emozionalmente i consumatori. L'utilizzo dell'allitterazione è piu frequente rispetto alle altre figure del suono: Aty ku toka takon qiellin Dajti Express; Ndani momentet e pranverës pa limite, në rrjetin më të mirë AMC; Një grup i fuqishëm në fushën e sigurimeve Sigal; Ju nuk mund ta blini lumturinë, por mund ta pini atë! Kakao Nesquik ecc.

Nei testi pubblicitari si ricorre spesso all'utilizzo di più figure retoriche che si combinano per aumentare l'effetto del messaggio ed assicurare una maggiore attenzione del pubblico. Nëse të duhet edhe pak më shumë..., ...sepse më shumë është më mirë! Dhe më mirë është $3 G$ nga Eagle Mobile!; E mban mend herën tënde të parë? Si asnjëherë tjetër....500 + 500minuta kombëtare+ltali + Greqi për ju të Dy Eagle Mobile; Joshës, tërheqës dhe jetëgjatë ... si dashuria e vërtetë Duel Celebration ecc.

Un altro meccanismo del linguaggio pubblicitario è l'adattamento creativo di proverbi, modi di dire o versi della letteratura in funzione della reclamizzazione del prodotto. La trasmissione del messaggio pubblicitario avviene attraverso una formulazione alternativa e nuova spesso divertente di enunciati già noti e cristallizati nell' uso che punta a soddisfare le esigenze di espressività, comprensibilità e complicità col consumatore: Dita e mirë fillon me më ngjes të mirë (dita e mirë duket që në mëngjes ); Një ofertë të tillë nuk do ta shohësh dy herë (jeta të jepet vetëm një herë) Albtelecom; Ta blesh apo të mos e blesh ? Alpha Realizo Kjo është përgjigjja! (Të rrosh a të mos rrosh ? Kjo është çështja); epo jeta është llogari more bir (epo jeta është matematikë Olimbi) llampa LED Optimal; Kthe diçka përmbys autocity;

A livello sintattico nel linguaggio della pubblicità si nota la prevalenza delle frasi brevi. In relazione all'informazione trasmessa negli slogan si predilige l'uso delle frasi imperative con l'impiego dell' imperativo che nella situazione comunicativa esprime la funzione pragmatica dell' invito o dell' esortazione rivolto al consumatore con lo scopo di coinvolgerlo nel compimento del contenuto proposizionale: Preke verën (Birra Tirana); Ndje ethet e Botërorit (Birra Stela ); Vishuni në Cacharel; Shijoje jetën! Celio; Jeto në maksimum! Dragon Heart; Jeto cdo moment! Pepsi; Gëzo kudo me çdo blerje! Banka Tirana; Zgjidhni bojën e duhur (bojra neon); Respekto edhe të gjallët! - Stop tek vijat e bardha!; Përjetoni shpejtësinë Abissnet; Përqafoje jetën! Spitali Amerikan; Shijoni botërorin 2014 në SUPER Sport!; Udhëto i sigurt! Atlantik; Shpërndaje Fjalën!Java Credins on line jepi vetes pushtet!; Credins on line merre bankën me vete !; Shënoni me interesat më të ulëta në treg Raiffaisen Bank; Jepini më shumë vlerë PASURISË tuaj! Sigal; Thuaj "Të dua" në mënyrën italiane me koleksionin e dashur të çokollatave Perugina.; Dhuroni disa Baci sot ! Baci Perugina; Ndani momentet e pranverës pa limite, në rrjetin më të mirë! Amc ecc .

Un altro tipo molto usato delle frasi semplici sono le frasi enuncative o dichiarative che nella pubblicità affermano un fatto o esprimomo un giudizio sempre positivo sul prodotto. Shkolla që sfidon të ardhmen Nobel; Albsig Ju kthen buzëqeshjen!; Abcom i kthen fuqinë telefonit fiks; Së bashku forcojmë shpirtin e skuadrës Societe Generale Albania; Zëri juaj është larg vetëm një telefonatë ; CREDINS bank Ne flasim gjuhën tuaj!; Jam ALBtelecom!; Alora i kthen qetësinë jetës tuaj!; Vetëm ne mendojmë për ju KMY. Të gjithë ne besojmë në KMY super; Lion Zgjon luanin tek ju! ecc.

Inoltre nella sintassi degli slogan pubblicitari si manifesta la preferenza per le frasi collegate per coordinazione di più frasi semplici. Ciascuna di esse costituendo una unità sintattica e semantica compiuta hanno senso compiuto anche se staccate l'una dall'altra. In questo modo ciascuna frase si può considerare come "minislogan": Jeto me Zemër dhe Ndaje Lumturinë! Algida; Analizojmë dhe përgjigjemi për jetën tuaj Intermedica; Risitë nuk i përzgjedhin njerëzit, ato na përkasin të gjithëve Digitalb ; Dhe gjithcka dua per festa je ti Birra Tirana; Clironi veten tuaj nga dhimbjet e mesit dhe kurseni 1990 lekë Cosmodisk; 20 vite së bashku dhe ky është vetëm fillimi Coca cola; Mjafton një telefonatë dhe dysheku vjen në shtratin tuaj Dormeo ecc.

La pubblicità mira a creare un rapporto di complicità con il pubblico usando un linguaggio che influenza fortemente il comportamento del consumatore. Questo spiega l' intensità con cui vengono utilizzati nella pubblicità i verbi nella prima e seconda persona del plurale e la frequenza dei pronomi personali e possessivi nella seconda persona singolare e plurale e nella prima persona singolare.

\section{II Panorama delle Lingue e i Valori di Riferimento nella Pubblicità Albanese}

1. Nella pubblicità destinata al pubblico albanese la lista delle lingue usate risulta variata. I dati raccolti dimostrano la presenza accanto all' albanese standard di alcune lingue straniere tra cui l'inglese, onnipresente nella società moderna; I' italiano, molto popolare in Albania per i stretti legami storici geografici e culturali con il paese di fronte l'Adriatico e il francese, una delle lingue straniere principali nel sistema dell' istruzione albanese.

L'albanese standard è la lingua usata nella comunicazione istituzionale di tutti i livelli, la lingua dell' istruzione e 
della diffusione dell' informazione dai media. Dall' analisi del corpus di dati raccolti risulta che l'albanese standard è inequivocabilmente la lingua della pubblicità rivolta al pubblico albanese ${ }^{4}$. II risultato è l'affermazione di quello che si percepisce anche direttamente dal flusso di pubblicità che ci arriva ogni momento da diversi mezzi di comunicazione ed è spiegabile col ruolo che ha l'albanese standard nel trasmettere il messaggio pubblicitario in modo chiaro e comprensibile a tutti i target di pubblico.

Tra le lingue straniere presenti nel paesaggio linguistico della pubblicità indirizzata al pubblico albanese l' inglese ha una frequenza molto più alta sia nei casi di compresenza di lingua albanese e lingua straniera, sia nei casi di presenza della sola lingua straniera. La prevalenza dell'inglese nella pubblicizzazione dei prodotti o servizi è determinata dalla sua diffusione come lingua dell' economia, della tecnologia e della comunicazione nell' epoca della globalizzazione in cui viviamo. L'uso di questa lingua nella pubblicità scritta e televisiva è una scelta mirata che corrisponde ad un triplice fine di conferire prestigio, affidabilità e notorietà internazionale al prodotto, di produrre l'effetto di coinvolgimento del consumatore e inoltre di assicurare la comprensione universale del messaggio trasmesso. L'impiego dell'inglese nel testo pubblicitario fa riferimento a diverse categorie merceologiche ( telefonia, alimentari, auto, casa sport ecc.) a prescindere dal paese d'origine del prodotto: Vodafone - Power to you; Have a break, have a Kit Kat; It's not scotch, It's not bourbon, It's jack; Nescafe Express Go...on; Smirnof -Yours for the making; Take the sky with u...Fiat; Peugeot - Let your body drive; Delta home - kitchen and sofas for friends; Eglo my light, my style; HikVision- Smart Evolution; Benetton- United colours of Benetton; Elle- love shoes; Albania, Go your own way!; With us, you just fly -It oil; Performance - Trasforming passion into excellence; fmtgroup energy power; Hardox wear plate; Bindi Media - Be all you can be... ecc.

La seconda lingua straniera presente nella pubblicità albanese è l'italiano. L'italiano a differenza dell'inglese è molto presente nella pubblicità scritta mentre è ridotto il suo uso in spot televisivi. L'impiego dell' italiano nel linguaggio pubblicitario si riferisce a categorie di servizi (banche, call center) e a categorie merceologiche (prodotti alimentari, prodotti per la casa, abbigliamento). La lingua italiana viene usata nelle pubblicità dei prodotti di marche italiane. Pasta Divella- Passione Mediterranea; Amadori-Passione di famiglia - La certezza di una grande marca; Morfeus- Il signore del sonno; Preferiamo i maschi perchè sono più competitivi; Facile.it - Scegliere è facile; La prealpina- stile italiano; Arreghini -stile e design, tecnologia e innovazione; Come ti piace il caffe? espresso, machiato, capuccino, freddo in tazza freda, irish - Kimbo espresso italiano ecc.

Nel linguaggio pubblicitario la presenza del francese rispetto alle altre lingue straniere è molto ridotta. I termini e le espressioni nella lingua francese sono presenti nelle pubblicità di categorie merceologiche quali le auto e prodotti di cosmetica Citroen Creative Tecnologie; La vie est belle Lancome.

2. L'analisi dei dati raccolti dimostra un panorama interessante in relazione ai valori di riferimento nella pubblicità albanese. II materiale pubblicitario vienne suddiviso in due gruppi ciascuno dei quali rimanda ad una di queste due tipologie di valori:

a) riferimento ai valori nazionali albanesi;

b) riferimento ai valori dei paesi europei ed extraeuropei.

La pubblicità televisiva e quella scritta che fa riferimento all'identità albanese e richiama i valori nazionali riguarda prodotti "Made in Albania". Jo, jo në botë s'ka, si birra Korça jonë in questa frase viene ribadita la qualità del prodotto ma anche l'essere un prodotto nostro, originario di Albania. Kmy Tradita shqiptare, lo spot sottolinea il fatto di essere una impresa che rispetta e porta avanti la tradizione albanese nella produzione di prodotti di salumeria. Birra Tirana Kuqalashe- $E$ kuqe pa turp, lo slogan del prodotto risalta il colore rosso caratteristico di questa birra che richiama il colore della bandiera, simbolo nazionale del popolo albanese mentre in un altro slogan dello stesso prodotto si rileva la sua unicità: Kuqalashe është vetëm një. Gëzuar i dashur mik, ju e meritoni të shijoni një verë si kjo! Vera bio Duka. Lo slogan del vino Duka evoca l'ospitalità del popolo albanese e la tradizione delle famiglie albanesi di ofrire agli ospiti le migliori pietanze e anche il miglior vino specificando la sua qualità bio. Questa linea pubblicitaria segue anche Gjirofarma che insiste sul legame esclusivo ed univoco dei suoi prodotti con il territorio dell' Argirocastro mettendo in evidenza la cultura e la storia locale nella produzione di latte e prodotti lattiero caseari, il rispetto per la tradizione, la naturalità dei prodotti e l'originalità del loro gusto che deriva dalla qualità del latte uttilizzato in quanto preserva l'essenza del territorio e il profumo della biodiversità floristica nel prodotto finale. Tek produktet e Gjirofarmës do të gjeni aspekte të kulturës lokale, historinë e prodhimit dhe respekt për traditën vendase; Gjiza Gjirofarm -Mjafton ta provosh dhe do të kesh ndjesinë e një ushqimi natyral...; Shijoni dhallën Gjirofarm, një produkt i një cilësie të lartë [ ...]duke ju ofruar shijen karakteristike të

\footnotetext{
${ }^{4}$ La presenza dei dialetti nella pubblicità albanese è isolata in casi sporadici. L' analisi dei dati dimostra che il materiale dialettale nella pubblicità coincide con la presenza negli spot di persone provenienti da zone diverse che raccontano al pubblico la loro esperienza positiva in relazione a un prodotto o serivizio offerto ( esull, kam ra nga pesha, e kum ba, gëzojna, kam përdor, s'do t'i nali , hithi, lumi kush mun me e ble, mjeri ai ecc.)
} 
dhallës ecc.

Quindi oggi si può parlare di prodotti nazionali che ci rappresentano e che la pubblicità albanese promuove. In alcuni casi anche le compagnie estere come Eagle Mobile, Vodafone, Coca cola calcano nei loro spot elementi distintivi dell'Albania e del popolo albanese (simboli come l' aquila e il colore rosso della bandiera nazionale, il carattere forte della gente, il rilievo montuoso del paese, l'orgolio nazionale per le radici antiche della popolazione e il patrimonio storico e culturale) per avvicinarsi sempre di più al pubblico, ai suoi valori ed alla sua mentalità. La pubblicità che evoca l'altra tipologia di valori riguarda prodotti che provengono da queste aree geografiche. Ci sono molti esempi di pubblicità in cui oltre le caratteristiche dei prodotti viene accentuato la loro provenienza come elemento di garanzia ma anche per sottolineare il legame esclusivo tra prodotto e paese d'origine: Altis marka nr. 1 e vajit të ullirit në Greqi; Babylino Sensitive Kontrolluar klinikisht në Institutin gjerman të dermatologjisë; Thuaj "Të dua" në mënyrën italiane me koleksionin e dashur të çokollatave Perugina; Conad Shija italiane pranë jush, o sono inseriti nella pubblicità elementi linguistici del paese d'origine del prodotto: Citroen Creative Tecnologie; Arreghini L'arte del colore diventa impresa; Amadori Passione di famiglia - La certezza di una grande marca.

\section{Conclusioni}

La pubblicità albanese a partire dagli anni '90 ha costruito progressivamente la notorietà di prodotti e marchi 0 ha promosso delle cause sociali contribuendo intanto a crescere se stessa e proporre una nuova forma di comunicazione. L'obbiettivo principale della pubblicità di convincere il pubblico ad acquistare il prodotto reclamizzato vienne affidato all' immagine giusta che stimola la curiosità e al linguaggio che garantisce l'efficacia del messaggio.

II linguaggio della pubblicità albanese presenta caratteristiche quali: il ricorso a soluzioni lessicali e sintattiche vicine a quelle del linguaggio ordinario, I' uso dei tecnicismi e delle lingue straniere in particolare dell' inglese, il riferimento ai modi di dire, l' uso consistente della seconda persona singolare e plurale per dare enfasi al pubblico e della prima persona per trasmettere il messaggio, l' uso consistente dell' imperativo e del presente dell' indicativo e la tendenza di ricorrere alle figure retoriche al fine di suggestionare il pubblico.

Nella lingua albanese mancano invece parole inventate per stuzzicare l' interesse del consumatore costruite sulla base di giochi di parole o dall'unione di frammenti di parole diverse (le parole macedonia). Nonostante questo la pubblicità ha il merito di aver fatto conoscere al pubblico una serie di lessemi nuovi ed ha concorso al loro passaggio nella lingua comune.

II messaggio pubblicitario vienne trasmesso al pubblico nell' albanese standard. Tuttavia si nota una presenza rilevante delle lingue straniere. Culturalmente la pubblicità albanese è riuscita nel corso degli anni ad esprimersi e suggestionare il pubblico facendo riferimento ai valori nazionali intrecciati con quelli universali nella promozione dei prodotti.

\section{Bibliografia}

Abruzzese A. (1991), Metafore della pubblicità, Genova, Costa e Nolan.

Baldini M. (1987),(a cura di), Il linguaggio della pubblicità, Roma, Armando.

Bellino F. (1991), Il linguaggio della pubblicità, Milano, Mursia.

Chiantera. (1989), Una lingua in vendita, L'italiiano della pubblicità, Roma,Carocci.

Galliot M. (1954), Essai sur la langue de la reclame contemporaine, Toulouse, Edouard Privat.

Giacomelli R. (2003), La lingua della pubblicità. In I. Bonomi, A. Masini, S.Morgana (a cura di), La lingua italiana e i mass media a cura, Roma, Carocci Editore.

Myers-Scotton C. (1993), Duelling Languages. Grammatical Structure in Codeswitching, Oxford, Clarendon Press.

Senes Grazia Maria (1981), L'inglese nella pubblicità alla radio-televisione e sulla stampa, in AA.VV.,Contatti interlinguistici e massmedia, Roma, La Goliardica.

Sergio G.(2008), La salute in vendita.Un sondaggio lessicale sulla lingua medico-pubblicitaria, in Lingua italiana d'Oggi, n.4, Roma, Bulzoni.

Thomai J.(2010), Leksikologji e gjuhës shqipe, Tiranë, Toena. 